\title{
Use of a shade guide to test the perception and prevalence of natural tooth colour in people of Nepal
}

\author{
Manu Rana', Sandeep Sharma² \\ ${ }^{1}$ Lecturer, Department of Conservative Dentistry and Endodontics, College of Medical Sciences, Bharatpur, ${ }^{2}$ Lecturer, \\ Department Of Oral Pathology, College of Medical Sciences, Bharatpur
}

Background: Recent advances in colour matching have been driven by the market demand for high-quality esthetic restorations. Improved shade guides, availability of shade-taking devices and research in the area of human colour vision have improved the potential of clinicians to achieve excellent colour matched restorations. A thorough understanding of appearance attributes of natural teeth is required along with these new tools to maximize shade matching results. Aims and Objectives: The aim of the present investigation is to determine tooth shade among the people of Nepal. Materials and Methods: In the present study, shade of cervical, middle and incisal third of the facial surface of natural maxillary central incisor was measured with digital colorimeter, VITA Easy shade Advance 4.0. It is an optical reading device that can assess the wavelengths reflected back to its sensors. Lighting in operatory does not affect the shade measurement. It is extremely accurate. The limitation of study was its small sample size. Result: The shade obtained in the cervical third of tooth is $1 \mathrm{M} 2(37.33 \%)$, in the middle $1 \mathrm{M} 1(32.88 \%)$, and in the incisal third $2 \mathrm{M} 1(37.33 \%)$. Value 1 indicates lighter tooth colour and colour saturation is up to 2. Conclusion: It was concluded that the most prevalent shade in cervical third was $1 \mathrm{M} 2$, middle third was $1 \mathrm{M} 1$ and incisal third was $2 \mathrm{M} 1$.

Key words: Tooth colour; Shade match; Digital colorimeter; VITA easyshade

\section{INTRODUCTION}

The vivid colours of nature infuse a dash of brilliance into the vibrant landscapes of life. These colours not only bring joy and brightness into our lives, but are elemental to all forms of beauty. In the field of dentistry, it forms an important basis for achieving superior esthetics. It is a challenge for every esthetic dentist to determine and replicate the appearance of teeth as it requires humility, patience and perseverance to mimic nature to its closest sense and form. Colour is governed by visual and scientific components and in dentistry, it is communicated on a regular basis but often misunderstood, since every human eye is not capable of perceiving it in a standardized manner. ${ }^{1}$

The oldest colour system was created by Albert $\mathrm{H}$. Munsellin 1905. It brought clarity to color communication by establishing an orderly system for accurately identifying every colour that exists. According to this, colour has three dimensions - hue $(\mathrm{H})$, value or lightness $(\mathrm{V})$, and chroma $(\mathrm{C})$. Hue is the dominant wavelength and helps to differentiate one color from another. Chroma is the saturation of color. Value is the darkness/lightness and is determined by the amount of black and white on a given scale. ${ }^{2}$ This was the first system to separate hue, chroma, and value into perceptually uniform and independent dimensions, systematically illustrating the colours in a three-dimensional space.

In 2001 by the Commission Internationale de 1 Éclairage (CIE), defined colour is: "The characteristic of visual perception that can be described through the attributes of Hue, Value and chroma. ${ }^{2}$ There are two ways of measuring tooth colour - subjective, through the use of shade guides, and objective, mainly through the use of electronic devices 
such as spectrophotometers, colorimeters and imaging systems. $^{3-6}$

The present study was designed to find the most prevalent shades in the Nepal population using VITA Easyshade $囚$ Advance 4.0.7,8

\section{MATERIAL AND METHOD}

The present study was based on measuring the shade of right maxillary central incisor of 225 volunteers from different areas of Nepal. As age affects the tooth colour perception, so in this study we chose only a particular age group, 18-25 years of age. Shade was measured by using a digital colorimeter, VITA Easyshade Advance 4.0.

\section{Inclusion criteria}

Vital and healthy maxillary central incisors were included in the study.

\section{Exclusion criteria}

Discoloured teeth, due to intrinsic or extrinsic stains, were not included.

- Intrinsic stains are those affecting the internal calcified tissues of the teeth, while extrinsic stains result from the deposition of a film, pigment, or calculus on the surface of enamel, exposed dentin, or cementum.

- Teeth with restorations do not represent the natural tooth surfaces and were not included.

- Caries result in decalcification and discoloration, so carious central incisors were excluded.

Before recording the shade of teeth, oral prophylaxis was done specially on central incisors and cleaned properly. To prevent cross contamination, the probe of VITA easy shade was covered with a disposable shield and calibration of the probe was done along with the shield. The probe tip was placed in contact with and at right angles to the labial surface first at cervical, then middle, and the incisal regions. The digital shade guide was set at a mode for taking reading at three points. Shade was measured at cervical, middle and incisal regions on the facial surface.

\section{RESULTS}

This study comprised 225 individuals, out of which 153 were males and 72 were female. According to the analysis of data, the most prevalent shade in cervical region was $1 \mathrm{M} 2$. It accounted for $37.33 \%$. This was followed by $1 \mathrm{M} 1$ which was $18.22 \%$. [Table 1 and Figure 1] dominant hue was $\mathrm{M}$ with lighter value.

In the middle third, the most prevalent shade was1M1 (32.88\%). This was followed by $2 \mathrm{M} 1$ (20\%).
Four teeth $(1.77 \%)$ had the lightest value, but chromatic saturation was 3 [Table 2 and Figure 2].

In the incisal third, the dominant hue was M. However, the value increased to 2. Maximum number of teeth (84) had shade in the range of 2M1. This was followed by $1 \mathrm{M} 1$. None of the teeth had a value darker than 2. Just two teeth were lighter with $0 \mathrm{M} 3$ shade [Table 3 and Figure 3].

\section{DISCUSSION}

Understanding the influence of factors such as age, gender and skin colour on selection of artificial teeth in completely edentulous patients with no pre-extraction records, presents a unique challenge to dentists all over the world. Smile has been said to be one of the most important

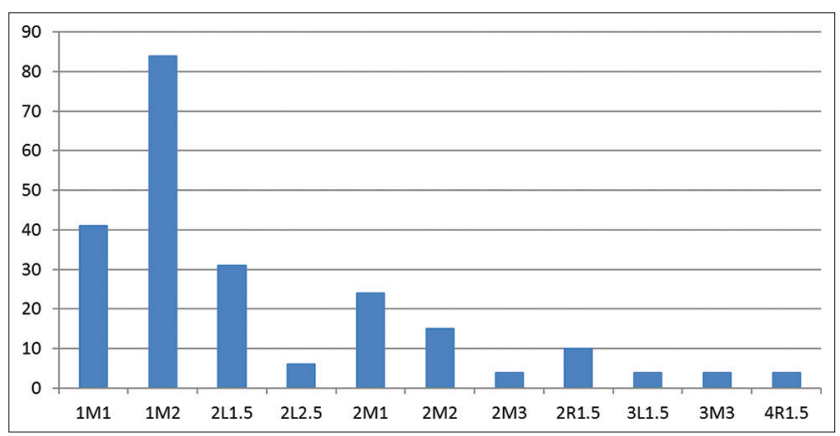

Figure 1: Graphical representation of shade observed at cervical third region of teeth

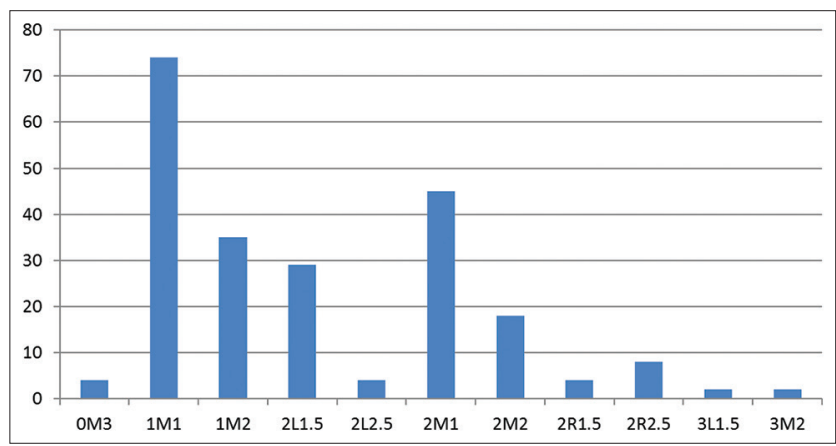

Figure 2: Graphical representation of shade observed at middle region of teeth

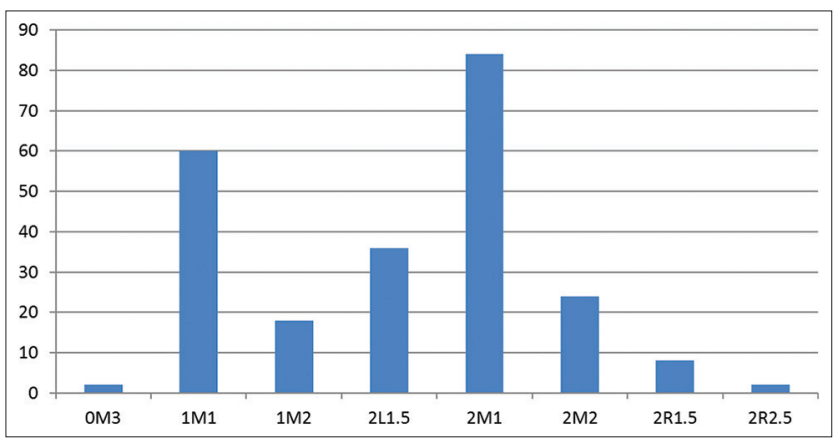

Figure 3: Graphical representation of shade observed at incisal third region of teeth

Asian Journal of Medical Sciences | Jan-Feb 2019 | Vol 10 | Issue 1 
Table 1: Shade at cervical third

\begin{tabular}{lccccccccccc}
\hline Shade & $\mathbf{1 M} \mathbf{1}$ & $\mathbf{1 M} \mathbf{2}$ & $\mathbf{2 L 1 . 5}$ & $\mathbf{2 L 2 . 5}$ & $\mathbf{2 M 1}$ & $\mathbf{2 M} \mathbf{2}$ & $\mathbf{2 M} \mathbf{3}$ & $\mathbf{2 R} \mathbf{1 . 5}$ & $\mathbf{3 L 1 . 5}$ & $\mathbf{3 M} \mathbf{3}$ & $\mathbf{4 R} \mathbf{5}$ \\
\hline Number of teeth & 41 & 84 & 31 & 6 & 24 & 15 & 4 & 10 & 4 & 4 & 4 \\
Percentage of shade & 18.22 & 37.33 & 13.77 & 2.66 & 10.66 & 6.66 & 1.77 & 4.44 & 1.77 & 1.77 & 1.77 \\
\hline
\end{tabular}

\begin{tabular}{|c|c|c|c|c|c|c|c|c|c|c|c|}
\hline Shade & 0M3 & $1 \mathrm{M} 1$ & $1 \mathrm{M} 2$ & 2L1.5 & $2 \mathrm{~L} 2.5$ & $2 \mathrm{M1}$ & $2 \mathrm{M} 2$ & $2 \mathrm{R} 1.5$ & $2 R 2.5$ & 3L1.5 & $3 \mathrm{M} 2$ \\
\hline Number of teeth & 4 & 74 & 35 & 29 & 4 & 45 & 18 & 4 & 8 & 2 & 2 \\
\hline Percentage of shade & 1.77 & 32.88 & 15.55 & 12.88 & 1.77 & 20 & 8 & 1.77 & 3.55 & 0.8 & 0.8 \\
\hline
\end{tabular}

\section{Table 3: Shade at incisal third}

\begin{tabular}{lcccccccc}
\hline Shade & 0M3 & 1M1 & 1M2 & 2L1.5 & 2M1 & 2M2 & 2R1.5 & 2R2.5 \\
\hline Number of teeth & 2 & 60 & 18 & 36 & 84 & 24 & 8 & 2 \\
Percentage of shade & 0.88 & 26.66 & 8 & 16 & 37.33 & 10.66 & 3.55 & 0.88 \\
\hline
\end{tabular}

interactive communication skills of a person and colour of teeth probably constitutes one of the most important parts of first impression of someone. However, there are authors who believe that when the overall dental appearance is considered, several factors are of significance, including tooth colour, shape, and position; restoration quality; and the general arrangement of the dentition.

The present study was designed to measure tooth colours of Nepal population so that it can help in shade selection for esthetic dental treatment. VITA Easyshade $\AA$ Advance 4.0 was used for measuring the shade as it is easy to use and reliable. The modal value selected was '3D Master' because of simplicity and easy to understand and has plenty of variability in shades to correspond the natural tooth colours.

The shade obtained in the cervical third of tooth is $1 \mathrm{M} 2(37.33 \%)$, in the middle 1M1 (32.88\%), and in the incisal third 2M1 (37.33\%). Value 1 indicates lighter tooth colour and colour saturation is up to 2 . However, the sample size in this study was small and represented only a limited range of age group. So there is need to conduct a study with larger and different age groups for promising results.

\section{CONCLUSION}

The conclusion drawn is that the most prevalent shade in the Nepalese population in the age group of 18-25 years is $1 \mathrm{M} 2$ in cervical, $1 \mathrm{M} 1$ in middle, and 2M1 in incisal. Out of the five value groups (1, lightest and 5, darkest), teeth are closer to the lighter value. The value increases from cervical to incisal. Within the yellow red range of natural tooth hues that is L (yellow) and R (red), the most frequent hue is M. Chroma is within 2 and decreases from cervical to incisal.

\section{REFERENCES}

1. Richiro R, Akirj S and Kanji I. Using a Computer Color-Matching System in Color Reproduction of Porcelain Restorations. Part 3: A Newly Developed Spectrophotometer Designed for Clinical Application. Int I Prosthodont 1994;7: 50-55.

2. Commission Internationale de l'Eclairage (CIE). Improvement tolndustrial Colour-Difference Evaluation. Vienna: CIE Central Bureau Public 2001; 142.

3. Joiner A. Tooth colour: A review of the literature. J Dent 2004; 32(1):3-12.

4. Brewer JD, Wee A and Seghi R. Advances in color matching. Dent Clin North Am 2004; 48: 341-358.

5. Cal E, Güneri $P$ and Kose T. Comparison of digital and spectrophotometric measurements of colour shade guides. J Oral Rehabil 2006; 33:221-228.

6. Boksman L. Shade selection; accuracy and reproducibility. Ont Dent 2007:24-27.

7. VITA Easyshade Advance 4.0 - The $4^{\text {th }}$ Generation Electronic Shade-Taking Device. Available from: http://www.vita-zahnfabrik. com

8. Posavec I, Prpić V and Zlatarić DK. Influence of light conditions and light sources on clinical measurement of natural teeth color using VITA easyshade advance $4,0 ®$ spectrophotometer. Pilot study. Acta Stomatol Croat 2016; 50:337-347.

9. Qualtrough AJ and Burke FJ. A look at dental esthetics. Quintessence Int 1994; 25:7-14.

\footnotetext{
Authors Contribution:

MR- Concept and design of the study, manuscript preparation, collected data; SS-Statistically analyzed and critical revision of manuscript.

Work attributed to: Department of Conservative Dentistry and Endodontics, College of Medical Sciences, Bharatpur.

Orcid ID:

Dr. Manu Rana- (1) https://orcid.org/0000-0002-9778-9831

Dr. Sandeep Sharma- idtps://orcid.org/0000-0001-7797-0273

Source of Support: Nil, Conflict of Interest: None.
} 